\title{
Audit of stroke care at the Institute of Neurology, National Hospital of Sri Lanka, 1994-1997
}

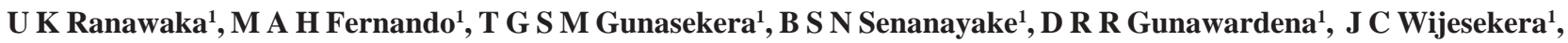 \\ and D J S Fernando ${ }^{2}$
}

(Index words: Retrospective case record evaluation, deficiencies, interventions)

\begin{abstract}
Objective To audit the process of stroke care.

Design Retrospective case record evaluation using an audit package designed by the Royal College of Physicians of London.

Setting Institute of Neurology, National Hospital of Sri Lanka, Colombo.

Patients 263 patients with stroke admitted over a period of 3 years.

Measurements Documentation of 60 audit items related to 13 aspects of stroke care.

Results The process of care was considered 'very good' for only $11(18.3 \%)$, and 'good' for only $9(15 \%)$ of the audit items. Care was 'average' for $5(8.3 \%)$, 'poor' for 9 $(15 \%)$ and 'very poor' for $26(43.3 \%)$ of the items.

Conclusions Stroke care was suboptimal in many aspects. Care related to rehabilitation oriented neurological assessments, initiation of secondary preventive measures, rehabilitation planning and discharge planning were especially deficient.

Competing interests: none declared. Some of the data reported in this paper have been presented at the Annual Scientific Sessions of the Sri Lanka Medical Association, 1998.
\end{abstract}

\section{Introduction}

Stroke is a much neglected illness. In spite of the enormous impact of stroke on patients, care givers and the society, stroke care remains poor throughout the world, even in developed countries (1). Stroke care in Sri Lanka has not been previously evaluated. We did an audit of stroke care at the Institute of Neurology of the National Hospital of Sri Lanka (NHSL), Colombo.

\section{Methodology}

\section{Patient selection}

Case records of all patients admitted to the Institute of Neurology over a period of 3 years (October 1994 to
September 1997) were scrutinised, and records of all patients with stroke $(n=263)$ were identified for retrospective analysis. No sampling was performed. Case selection was based on the clinical diagnosis of stroke based on WHO criteria (2), and recorded confirmation by computed tomography (CT) where available. Cases with an initial clinical diagnosis of stroke, all subsequently refuted by CT were excluded. Patients with transient ischaemic attacks (TIAs) and patients readmitted for further investigation or management of complications of a previous stroke were also excluded.

\section{Audit package}

An audit package designed by the Royal College of Physicians of London (RCP) and the United Kingdom Stroke Audit Group (3) was used to evaluate the process of stroke care. The package consisted of stroke audit forms for the data entry, and software for data analysis. The RCP stroke audit package has been previously used in many centres $(4,5)$.

\section{Data collection}

Data from the case records were recorded in $\mathrm{RCP}$ stroke audit forms and trasferred into a computer database. The audit form contained 60 audit items, categorised under 13 subheadings assessing different aspects of documentation of stroke care (Table).

There were 3 possible responses to each audit item; 'Yes' - if the information has been recorded, or 'No' - if the information has not been recorded, or 'No, but' - if the information has not been recorded, but this was considered acceptable in the given circumstance. For example, not assessing visual fields or speech in an unconscious patient would be recorded as a 'No, but' response. The relevant information had to be recorded within a specified time frame to be considered acceptable.

\section{Data analysis}

The result for each audit item was expressed as a percentage value $(\mathrm{OK} \%)$, which was an indication of the quality of documentation of care.

${ }^{1}$ Institute of Neurology, National Hospital of Sri Lanka,Colombo. ${ }^{2}$ Faculty of Medical Sciences, University of Sri Jayawardenapura, Nugegoda, Sri Lanka. (Received 5 January 2002; revised version accepted 31 May 2002. Corresponding author UKR, telephones +94 1 674681, +94 1 91790, email: udayaran@yahoo.com). 


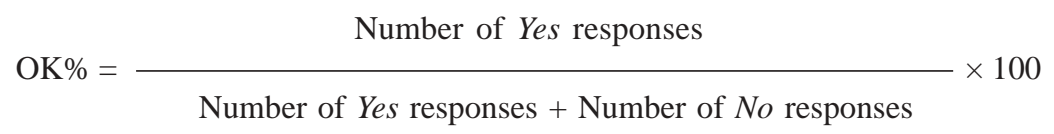

For the purpose of this study, we graded quality of care according to the OK\% as follows:

very good (81 to $100 \%)$, good (61 to $80 \%$ ), average (41 to $60 \%$ ), poor (21 to $40 \%$ ), and very poor (0 to $20 \%$ ).

Table. Documentation of audit items of stroke care

Audit item

\section{History}

Source of history

Rate of onset of symptoms

Record of admission drugs

2. Risk factors

Previous stroke or TIA

History of hypertension

History of heart disease

History of diabetes

Peripheral vascular disease

History of hyperlipidaemia

Smoking (32), alcohol (30)

3. Pre-stroke function

Record of dependence

Use of social services

Employment

\section{General examination}

Pulse rate and rhythm (42), blood pressure (96), heart sounds (79), Neck bruits (22), peripheral pulses (2), funduscopy (89)

\section{Neurological examination}

Conscious level (82), eye movements (69), power in the limbs (94), communication (39), trunk control or gait (12), swallowing (20), formal mental test score (2), visuospatial function (2), visual fields (36), sensory testing (58)

\section{Clinical diagnosis}

Clear diagnostic formulation

$\mathrm{OK} \%$

82

19

OK\% - quality of care, expressed as a percentage (see text) Values within parenthesis are percentages
Audit item

$\mathrm{OK} \%$

7. Usual baseline investigations Full blood count (93), ESR (73), Urea and electrolytes (89), Glucose (97), ECG (62)

\section{Special investigations}

CT Scan

Investigation for rare causes

9. Immediate management plan

Hydration

Urinary incontinence

10. Management in the first week

Consultant review

Review of important deficits

\section{Rehabilitation}

Record of personal interests, list of patients' problems, objectives or rehabilitation goals, re-assessment of functional status, multidisciplinary meeting, information given to patient/relatives - all $<4 \%$

\section{Discharge planning}

Ownership of accommodation, type of accommodation, living alone/not, stairs/ ground floor / lift, access to toilet, informal support available - all $<3 \%$

\section{Secondary prevention}

Blood pressure after 4 days

Antihypertensive medication

Long term aspirin

Long term anticoagulation

Advice about smoking

Non-invasive carotid imaging 


\section{Results}

'Very good' care as defined for the study was achieved only in $11(18.3 \%)$ of the audit items. These included certain neurological assessments (such as recording of symptom evolution, blood pressure, conscious level, limb power, funduscopy), performing baseline investigations (such as blood count, urea and electrolytes, blood glucose), and consultant review. 'Good' care was noted for only 9 (15\%) of the audit items. Carrying out routine investigations such as erythrocyte sedimentation rate (ESR) and ECG, computed tomography (CT) scanning, investigating for rare causes of stroke and starting aspirin for secondary prophylaxis were among these.

Care was 'average' for 5 (8.3\%), 'poor' for 9 (15\%) and 'very poor' for $26(43.3 \%)$ of the items. Basic clinical data such as drug history, previous strokes or TlAs, peripheral vascular disease, smoking and alcohol habits, and cardiac rate and rhythm were poorly documented. Assessment of aspects important in rehabilitation such as pre-stroke function, communication, trunk control or gait, swallowing, visuospatial function and visual fields was extremely unsatisfactory. Formulation of a clear and complete diagnosis, and immediate management of hydration and urinary incontinence were deficient. Many aspects related to rehabilitation planning, discharge planning and secondary prevention were very poor (Table).

\section{Discussion}

We used a previously validated audit instrument, the RCP audit package, for this study. The RCP stroke audit was simply an audit of good clinical practice. The majority of the items audited (51 out of 60) are basic clinical variables that can be easily assessed by the bedside, and the routine investigations are readily available in most hospitals. Facilities for CT scanning, non-invasive carotid imaging and investigation for unusual causes of stroke (echocardiography, haematological screening etc.) were available at the NHSL during the period of study. The RCP audit package was therefore applicable in our setting.

Audit is a powerful tool for evaluating patient care in clinical practice, enabling identification of relative strengths and weaknesses. This study indicated serious deficiencies in stroke care at the Institute of Neurology. Rehabilitation oriented clinical assessments, rehabilitation planning and discharge planning were extremely poor. This may have been partly due to the paucity of comprehensive rehabilitation services in acute care settings, the complete absence of a multidisciplinary approach to patient care, and the lack of emphasis on rehabilitation in undergraduate and postgraduate medical education.

The purpose of audit is to identify deficiencies of care, and to introduce remedial interventions. As a result of this audit, several changes were made in the structure and process of stroke care at the Institute of Neurology. These included the introduction of a structured clerking format to improve documentation, including rehabilitation oriented neurological and functional status assessments. A widely used functional assessment scale, the Barthel Index of activities of daily living (6), was included in the clerking format. A stroke unit was established at the Institute. A multidisciplinary stroke team was set up, with specialist inputs in speech therapy, physiotherapy, occupational therapy and social services support.

\section{Conclusion}

The RCP stroke audit package was successfully used at the Institute of Neurology, and stroke care was found to be suboptimal in many aspects. The audit has served to bring about major changes, which we believe will lead to significant improvements in stroke care.

\section{References}

1. Fifth King's Fund Forum: Consensus statement. Treatment of stroke. British Medical Journal 1988; 297: 126-8.

2. Bonita R. Epidemiology of stroke. Lancet 1992; 339: 342-7.

3. Royal College of Physicians. Stroke Audit Package. London: Royal College of Physicians, 1994.

4. Hancock RJT, Oddy M, Saweirs WM, Court B. The RCP audit package in practice. Journal of the Royal College of Physicians of London 1997; 31: 71-8.

5. McNaughton H. Stroke audit in a New Zealand hospital. New Zealand Medical Journal 1996; 109: 257-60.

6. Mahoney FI, Barthel DW. Functional evaluation: the Barthel Index. Maryland State Medical Journal 1965; 14: 61-5. 